\title{
RNase MRP RNA and human genetic diseases
}

\author{
Allison N Martin ${ }^{1}$, Yong $\mathrm{Li}^{1}$ \\ ${ }^{1}$ Department of Biochemistry and Molecular Biology, and Center for Genetics and Molecular Medicine, School of Medicine, University \\ of Louisville, 319 Abraham Flexner Way, Louisville, KY 40202, USA
}

RNase MRP RNA is the RNA subunit of the RNase mitochondrial RNA processing (MRP) enzyme complex that is involved in multiple cellular RNA processing events. Mutations on RNase MRP RNA gene (RMRP) cause a recessively inherited developmental disorder, cartilage-hair hypoplasia $(\mathrm{CHH})$. The relationship of the genotype (RMRP mutation), RNA processing deficiency of the RNase MRP complex, and the phenotype of CHH and other skeletal dysplasias is yet to be explored.

Keywords: RNase MRP RNA, cartilage-hair hypoplasia

Cell Research (2007) 17:219-226. doi: 10.1038/sj.cr.7310120; published online 26 December 2006

\section{Introduction}

There are over 17000 human genetic disorders listed in the database Online Mendelian Inheritance in Man (OMIM) [1]. The vast majority of them relate to proteincoding genes, whereas only a few noncoding RNA genes have been linked to genetic diseases. Noncoding RNA is an RNA molecule that functions without being translated into a protein. In this review, we will summarize recent advances in the understanding of one noncoding RNA, including the physiological roles of its functional complex and its association with various inherited skeletal dysplasias.

\section{The function of RNase MRP}

The RNase MRP complex (MRP stands for mitochondrial RNA processing) is a eukaryotic-specific ribonucleoprotein originally identified in murine cells by virtue of its ability to process an RNA transcript complementary to the light strand to generate RNA primers for the heavy-strand DNA replication in vitro (Figure 1A) [2]. Biochemical purification of the RNase MRP activity from mitochondria showed that this enzyme is a ribonucleoprotein that contains one RNA subunit (MRP RNA), a nuclear gene product, which is essential for catalysis [2]. In addition to its mitochondrial

Correspondence: Yong Li

Tel: +1-502-852-7551; Fax: +1-502-852-6222;

E-mail: yong.li@louisville.edu function, evidence for the involvement of the RNase MRP complex in the biogenesis of ribosomes has been obtained in the yeast Saccharomyces cerevisiae [3, 4]. RNase MRP was demonstrated to cleave the precursor of ribosomal RNA (rRNA) at the A3 site within the internal transcribed spacer 1 in S. cerevisiae (Figure 1B). This cleavage produces both a long form and a short form of 5.8S rRNA under wild-type conditions; however, if a mutation has occurred that changes the activity of RNase MRP, the long form of 5.8S rRNA is shown to be accumulated due to the inhibition of cleavage at site A3. Subcellular partitioning and in situ hybridization experiments confirmed the presence of RNase MRP in mitochondria and nucleoli, with the vast majority being localized in the nucleolus [5]. The central region (118-175 nt; Figure 2) of the RNase MRP RNA is responsible for its localization in mitochondria, whereas the $\mathrm{Th} / \mathrm{To}$ (namely, the Rpp38 protein subunit) binding site, located at the 5'-terminal region of the RNase MRP RNA, is responsible for the nucleolar localization of the complex [6]. Genetic depletion of either the RNA or protein components of $S$. cerevisiae RNase MRP demonstrated that the RNase MRP complex is essential for cell viability [7]. RNase MRP is also implicated in messenger RNA (mRNA) degradation and cell cycle progression at the end of mitosis. It processes the 5'-UTR of the cyclin B2 (CLB2) mRNA and regulates the completion of mitosis $[8,9]$.

\section{RNase MRP RNA and cartilage-hair hypoplasia}

Approximately 40 years ago, some members of the Old 


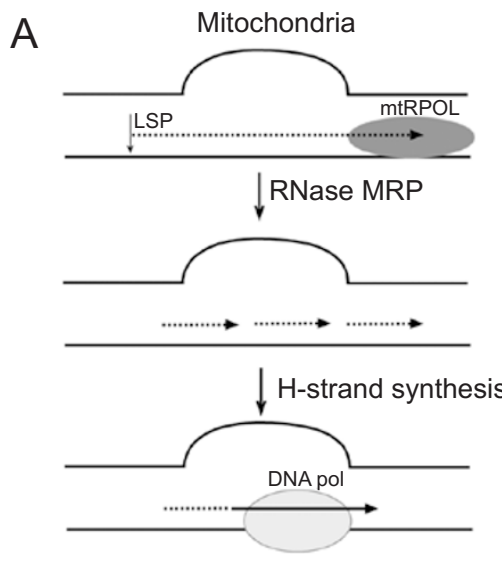

C

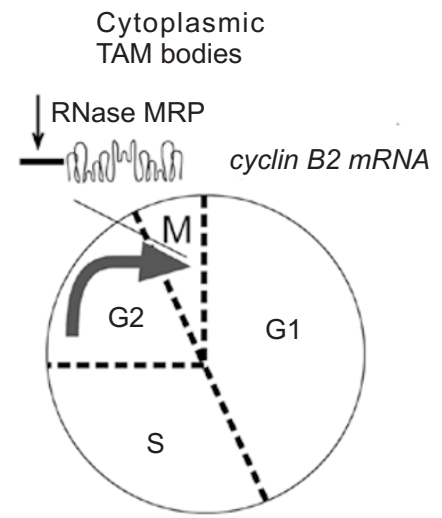

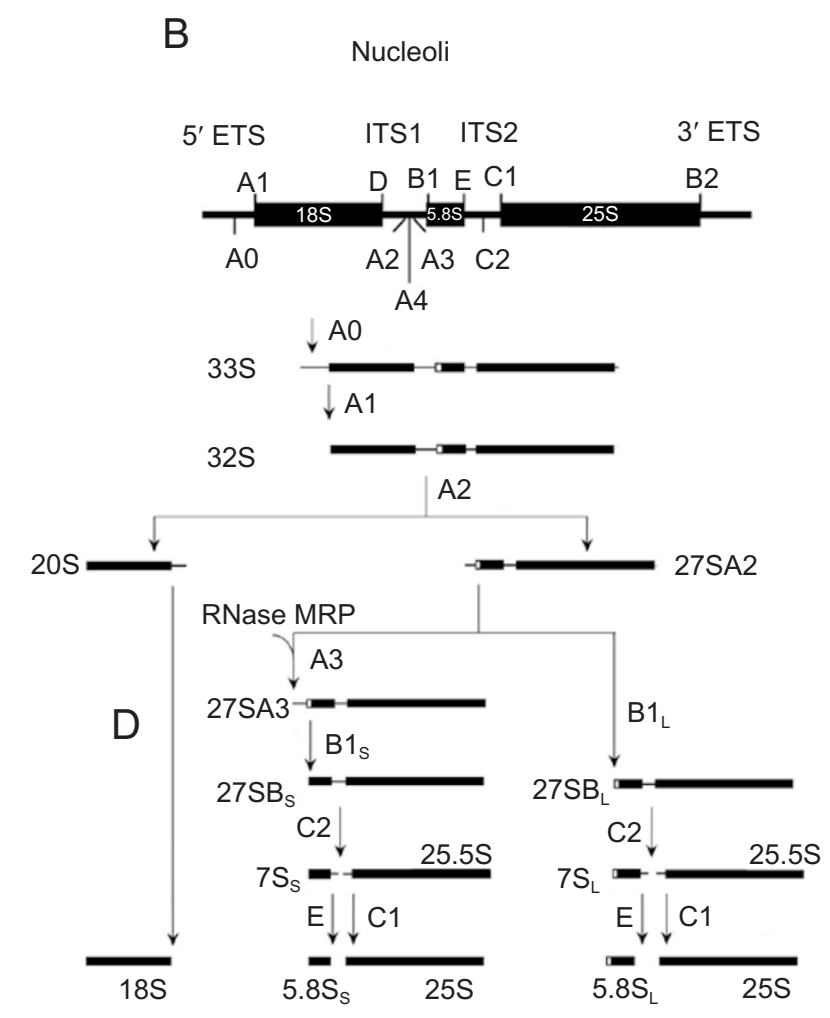

$18 S$
B

5' ETS

335

A2

Figure 1 Function of the RNase MRP enzyme complex. (A) RNase MRP is involved in the processing of mitochondrial RNA that functions as a primer for mitochondrial DNA replication in mitochondria. Transcription starts from the light-strand promoter by mitochondrial RNA polymerase. After transcription of the heavy-strand origin of replication, the transcript remains bound to the DNA duplex and is cleaved by RNase MRP to form primers that are used for the initiation of DNA synthesis by DNA polymerase $\gamma$ [29]. (B) RNase MRP functions in the pre-rRNA processing in $S$. cerevisiae in nucleoli. The $35 \mathrm{~S}$ primary transcript is processed into mature 25S, 18S, and 5.8S rRNAs [30]. The cleavage sites (A0 through E), the external transcribed spacers (5'-ETS and 3'-ETS), and the internal transcribed spacers (ITS1 and ITS2) are indicated. The small white box marks the sequence in the long form of 5.8S rRNA, $5.8 \mathrm{~S}_{\mathrm{L}}$, that is absent in the short form, $5.8 \mathrm{~S}_{\mathrm{S}}$. RNase MRP processes the A3 site in ITS1 [3, 4]. (C) RNase MRP processes the 5'-UTR of CLB2 mRNA in cytoplasmic temporal asymmetric MRP (TAM) bodies. CLB2 mRNA normally disappears rapidly as cells complete mitosis. RNase MRP mutations have an exit-from-mitosis defect and a late anaphase delay. RNase MRP specifically cleaves the CLB2 mRNA in its 5'-UTR to allow rapid 5' to 3' degradation by the Xrn1 nuclease. Degradation of the CLB2 mRNA by RNase MRP provides a novel way to regulate the cell cycle that complements the protein degradation machinery [31].

Order Amish community in Pennsylvania were found to be suffering from an unusual inherited disease [10]. Later, the disorder was also discovered in a small fraction of the Finnish population [11]. The carrier frequencies among the Amish and the Finns are 1:19 and 1:76, respectively [11]. People with the disease exhibit the short-limbed dwarfism due to skeletal dysplasia, have changes in the structure and abundance of their hair (hypoplastic hair), and show abnormalities in the development and function of their bone and cartilage. For these reasons, Dr Victor McKusick named the disease as cartilage-hair hypoplasia $(\mathrm{CHH})[10]$, which is also known as metaphyseal chondrodysplasia (MCD) McKusick type (OMIM, \#250250). To identify which gene or genes carry mutations in people with $\mathrm{CHH}$ is a daunting task [12]. Yet after years of genetic mapping and DNA sequencing, the responsible gene was found to be a 265-nt noncoding RNA gene [RNase MRP RNA gene $(R M R P)]$ encoding the human MRP RNA [12]. This was the first nuclear noncoding RNA gene to be found to cause a genetic disease. 


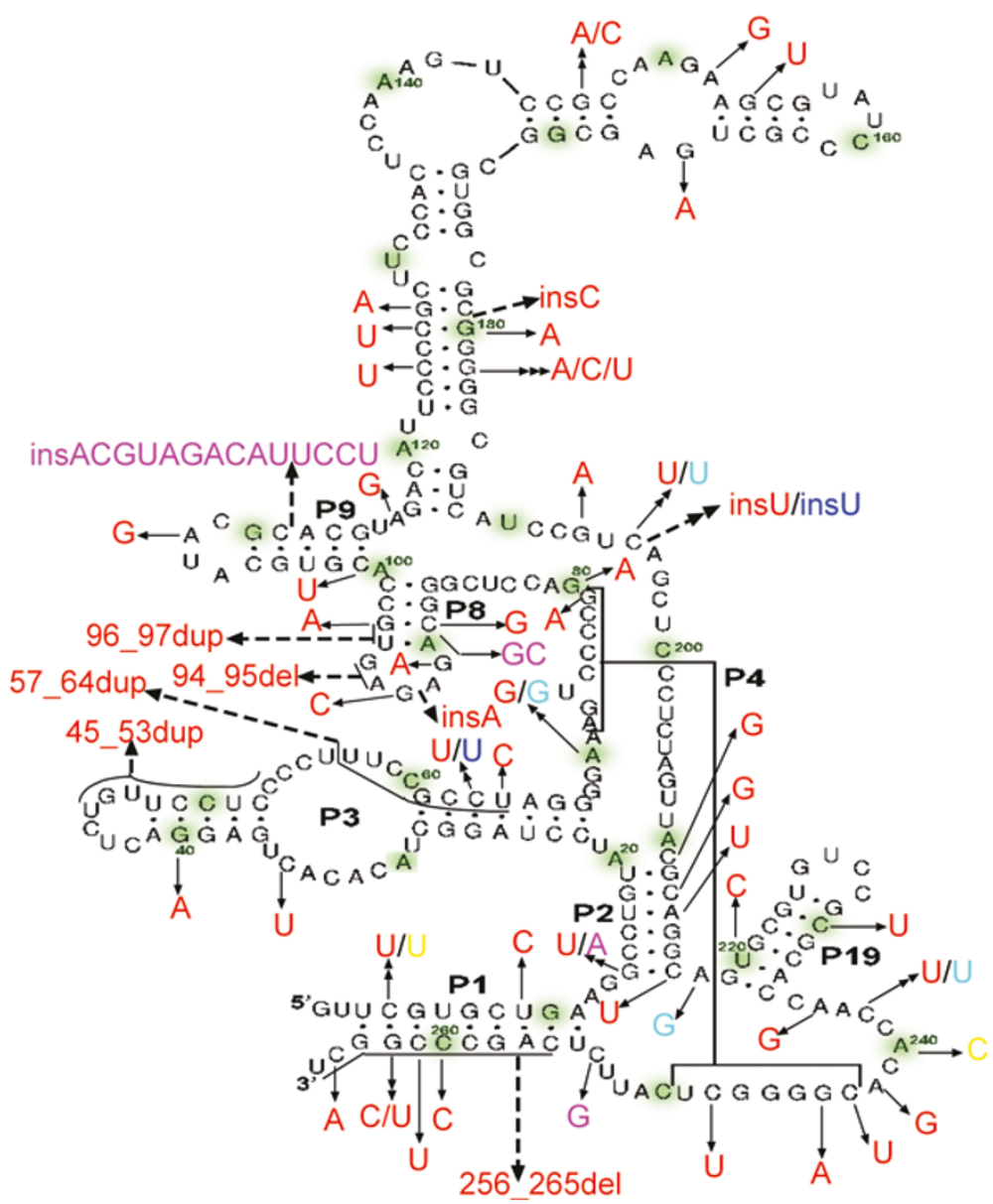

Figure 2 The secondary structure of RNase MRP RNA with mutations in human genetic disease. The RNA sequence is deduced from the RMRP gene M29916 of GenBank. Unique mutations for CHH are shown in red, kyphomelic dysplasia in blue, AD in light magenta, MDWH in cyan, and Omenn syndrome in yellow. $4 \mathrm{C} \rightarrow \rightarrow \mathrm{U}$ (red)/U (yellow) denotes that the $4 \mathrm{C} \rightarrow \mathrm{U}$ mutation occurs in both $\mathrm{CHH}$ and Omenn syndrome, whereas $182 \mathrm{G} \rightarrow \mathrm{A}(\mathrm{red}) / \mathrm{C}(\mathrm{red}) / \mathrm{U}$ (red) denotes that $182 \mathrm{G}$ can be mutated to $\mathrm{A}, \mathrm{C}$, or $\mathrm{U}$ in $\mathrm{CHH}$ patients. Mutations in the promoter region between the TATA box and transcription initiation site are not included in this figure (see Table 1).

Mutations leading to $\mathrm{CHH}$ are predominantly found in both the transcribed region and the promoter region (from the TATA box to the transcription initiation site) (Table 1) of the RMRP gene. The most frequently found mutation is the $70 \mathrm{~A} \rightarrow \mathrm{G}$ point mutation [11-17]. Other mutations that have been recorded in patients with $\mathrm{CHH}$ are summarized in Table 1. Insertional mutations and duplications in the upstream region of the $R M R P$ gene, between the TATA box and the point of transcription initiation, were shown to silence transcription [18]. Some mutations in the transcribed region of the gene, which may negatively affect transcriptional elongation, are also shown to cause impedance of transcription $[12,18]$. It is interesting to note that no patient has been found to be homozygous or compound heterozygous for the promoter region duplica- tions/insertions, indicating that such mutations may be phenotypically lethal [19].

Bonafé et al. [19] reported a series of 20 novel RMRP mutations in patients with $\mathrm{CHH}$ and summarized all the putative pathogenic mutations and single nucleotide polymorphisms (SNPs). They found that putative pathogenic mutations of the transcribed regions of the $R M R P$ gene are located in regions of highly conserved nucleotide sequences, whereas SNPs without phenotypic consequences are located in areas of non-conserved nucleotides. They also aligned the promoter region of the gene from various mammalian species and found a similar pattern: $\mathrm{CHH}$-associated mutations occur in regions of strongly conserved nucleotide sequences, whereas non-pathogenic SNPs mainly occupy areas of non-conserved nucleotides of the promoter region [19]. 
Table 1 Mutations in the RMRP

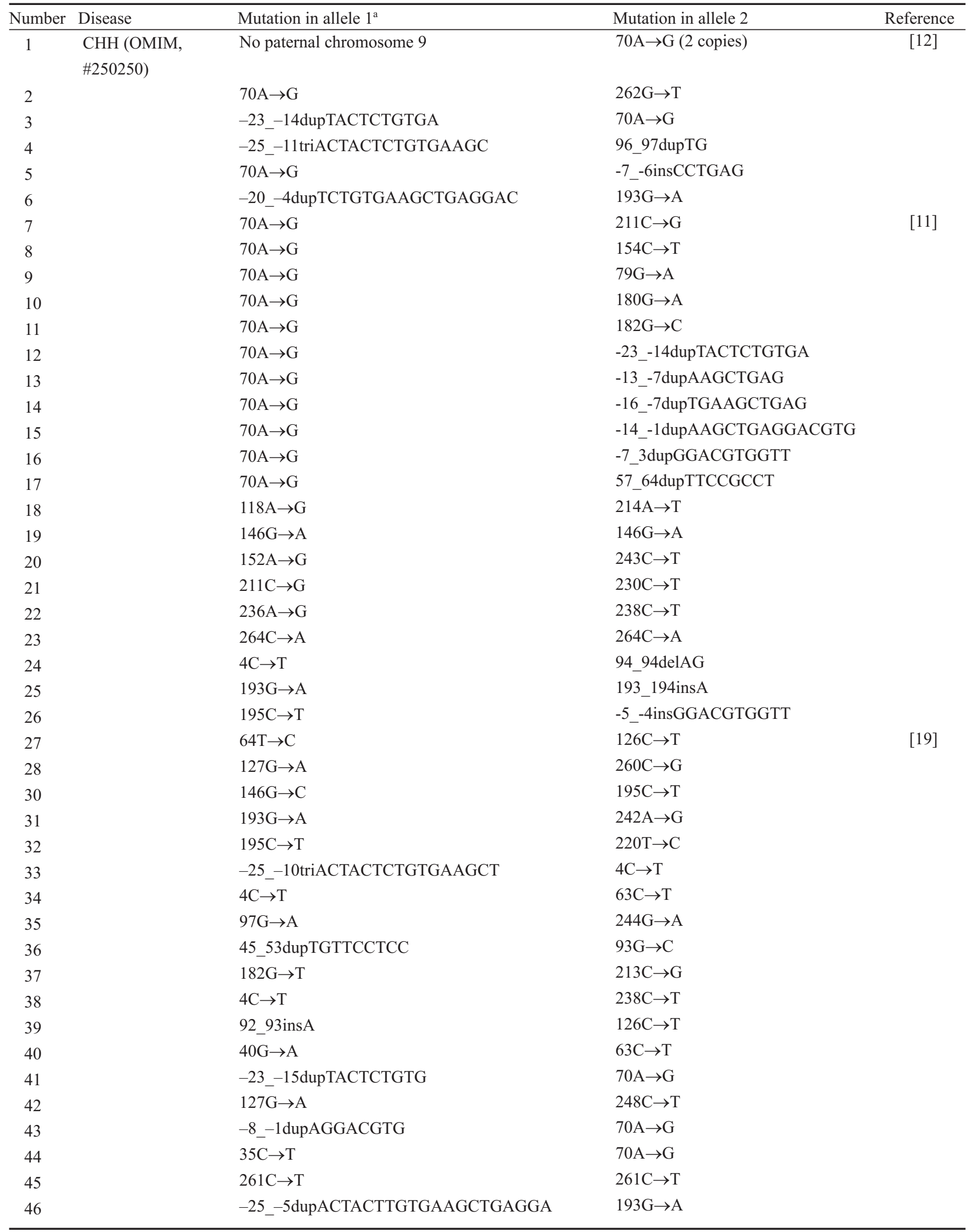


Table 1 Mutations in the RMRP (continued)

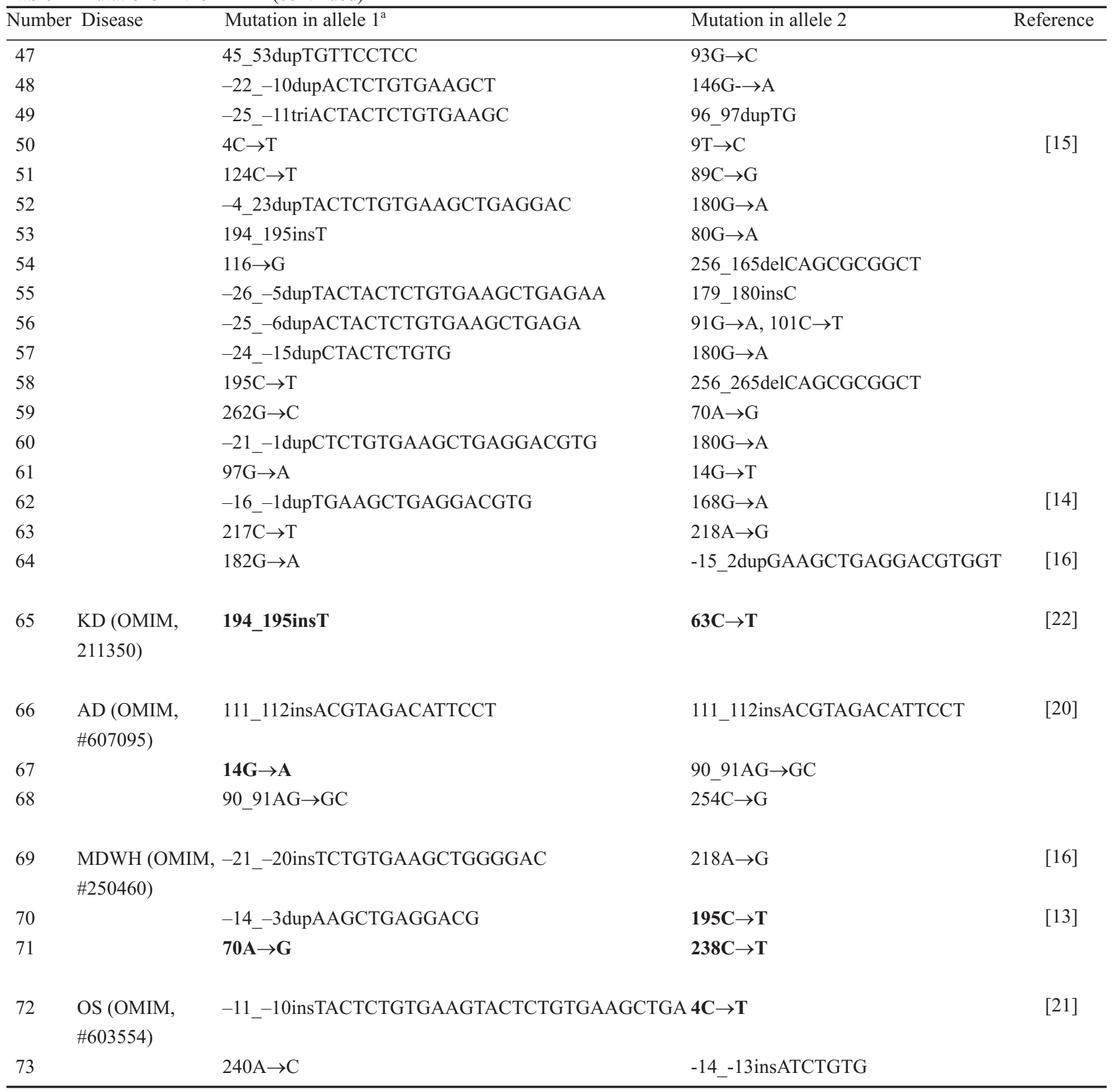

${ }^{a}$ Abbreviations: AD, anauxetic dysplasia; KD, Kyphomelic dysplasia; $\mathrm{CHH}$, cartilage-hair hypoplasia; OS, Omenn syndrome; dup, duplication; ins, insertion; MDWH, metaphyseal dysplasia without hypotrichosis; OMIM, Online Mendelian Inheritance in Man; tri; triplication. This table is abridged with the following consideration: mutations that are listed in alleles of other patients will not be recorded unless at least one allele has a novel mutation. For example, if we have mutations of $70 \mathrm{~A} \rightarrow \mathrm{G} / 262 \mathrm{G} \rightarrow \mathrm{T}$, we will list $70 \mathrm{~A} \rightarrow \mathrm{G} / 195 \mathrm{C} \rightarrow \mathrm{T}$, but not $70 \mathrm{~A} \rightarrow$ $\mathrm{G} / 70 \mathrm{~A} \rightarrow \mathrm{G}$ or $262 \mathrm{G} \rightarrow \mathrm{T} / 70 \mathrm{~A} \rightarrow \mathrm{G}$. Mutations that occur in more than one disease are shown in bold.

\section{Other diseases}

Beyond CHH, RNase MRP RNA mutations have been implicated in a variety of other inherited diseases, including metaphyseal dysplasia without hypotrichosis (MDWH), anauxetic dysplasia (AD), kyphomelic dysplasia (KD), and Omenn syndrome (OS).

MDWH (OMIM, \#250460) is a disorder in which the 
metaphyses of long bones fail to produce normal new tubular structures and instead appear to be expanded and porous [16]. Hypotrichosis is simply defined as having a less than normal amount of hair. MDWH lies within a group of heterogeneous skeletal dysplasias called MCD. Patients with MDWH display several unique and heterozygous mutations on the RMRP gene. One of these distinctive mutations is an insertion (-21_-20insTCTGTGAAGCTGGGGAC ) on the paternal allele, with a $218 \mathrm{~A} \rightarrow \mathrm{G}$ point mutation on the maternal allele (Table 1). MDWH is similar to $\mathrm{CHH}$ in terms of short stature metaphyseal dysplasia; yet, it lacks hair anomaly, immunodeficiency, and other extra skeletal features of $\mathrm{CHH}$. Bonafé et al. [19] reported two more cases of $\mathrm{MDWH}$, of which the mutations $(70 \mathrm{~A} \rightarrow \mathrm{G}, 195 \mathrm{C} \rightarrow \mathrm{T}$, and $238 \mathrm{C} \rightarrow \mathrm{T}$ ) also exist in some $\mathrm{CHH}$ patients (Table 1 ). Thus, it is likely that MDWH is a variant of $\mathrm{CHH}$.

$\mathrm{AD}$ (OMIM, \#607095) is an autosomal recessive spondylometaepiphyseal dysplasia and is characterized by prenatal onset of extreme short stature and an adult height of less than $85 \mathrm{~cm}$. Additionally, the disease commonly includes hypodontia, which is defined as having less than the normal number of teeth, and mild mental retardation [20]. RMRP gene mutations were once again implicated in the occurrence of this disease, which is allelic to $\mathrm{CHH}$ and MDWH. The sequencing of the $R M R P$ gene in patients with AD showed one homozygous insertion mutation, 111_112insACGTAGACATTCCT, and two compound heterozygous mutations, $+14 \mathrm{G} \rightarrow \mathrm{A} / 90 \_91 \mathrm{AG} \rightarrow \mathrm{GC}$ and $90 \_91 \mathrm{AG} \rightarrow \mathrm{GC} / 254 \mathrm{C} \rightarrow \mathrm{G}$ (see Table 1) [20].

OS (OMIM, \#603554) is a severe immunodeficiency disease that is most commonly characterized by generalized scaly exudative erythroderma, which is an intense and usually widespread reddening of the skin and is often associated with exfoliation of the skin. OS is usually accompanied by enlarged lymphoid tissues, protracted diarrhea, failure to thrive, and eosinophilia, which refers to the condition characterized by the presence of high amounts of eosinophils in either the blood or body tissues [21]. Mutations in the nucleases encoded by the recombination activating genes ( $R A G 1$ and $R A G 2$ ) or in the Artemis gene were found in some but not all patients with OS. It has been suggested in recent literature that secretion of some T-cell clones in the blood of patients with OS is largely responsible for the phenotype of the disease. Treatments with either cyclosporine or corticosteroids before bone marrow transplantation can alleviate some of the symptoms of OS [21]. Roifman et al. [21] have examined two patients who exhibited clinical features consistent with OS but had no mutations in $R A G$ or Artemis genes. Sequence analysis of the $R M R P$ gene revealed three novel mutations, -11 10insTACTCTGTGAAGTACTCTGTGAAGCTGA/4C $\rightarrow$ $\mathrm{T}$ (identified previously in $\mathrm{CHH}$ patients) for the first patient and $240 \mathrm{~A} \rightarrow \mathrm{C} /-14$ - 13 insATCTGTG for the second one. These results indicate that mutations in the RNase MRP may also be associated with OS.

$\mathrm{KD}$ (OMIM, \#211350) is a type of short-limbed dwarfism and skeletal dysplasia characterized by the bowing of long bones, normal intelligence, disproportionate growth, mild facial dysmorphia, flattened vertebrae, and short ribs [22]. Although KD has only been observed in a small number of patients, this sublethal disease remains relevant to discussions of the distinct manifestations of MCD. KD is quite similar to other forms of MCD in that it exhibits combined immune deficiency and aplastic anemia. Femoral bowing is a hallmark of KD [23]. Novel mutations were found in the $R M R P$ gene of a patient with $\mathrm{KD}$, including an insertion of T at 194_195 (paternal allele) and a 63C $\rightarrow$ $\mathrm{T}$ point mutation on the maternal allele. Both mutations are also found in $\mathrm{CHH}$ patients (Table 1 and Figure 2).

\section{CHH-related RNA metabolism}

It has been speculated that hypoplastic anemia of $\mathrm{CHH}$ is related to the ribosome biosynthesis function of the RNase MRP enzyme (Figure 1B). Interestingly, several genetic disorders associated with bone marrow failure are found to exhibit molecular defects in ribosome biogenesis. Beyond RMRP (pre-rRNA processing) in $\mathrm{CHH}$, other genes/diseases linked to ribosomal biosynthesis include $D K C 1$ (rRNA modification) in X-linked dyskeratosis congenital, RPS19 (40S ribosomal subunit maturation) in Diamond-Blackfan anemia, and SBDS (40S ribosomal subunit maturation) in Shwachman-Diamond syndrome. RPS19, one of the protein components of 40S small ribosomal subunit, is involved in the interaction of the $40 \mathrm{~S}$ subunit and elongation factor 2 (eIF-2) [24]. A 5.8S rRNA mutant was demonstrated to substantially reduce the level of eIF-2 associated with polyribosomes [25]. These results suggest the potential connection of RPS19, 5.8S rRNA and, to certain degree, the biological function of RNase MRP, although there is no direct evidence for overproduction of the long non-natural form of 5.8S rRNA (Figure 1B) in $\mathrm{CHH}$ patients as that in yeast (Figure 1B). However, the role of ribosome biosynthesis in the pathogenesis of $\mathrm{CHH}$ has been questioned by recent studies. By comparing the phenotype of $\mathrm{CHH}$ with that of a similar disease, $\mathrm{AD}$, it has been observed that hypoproliferative bone marrow dysfunction and the tendency to develop cancer (mostly lymphoma) are observed only in $\mathrm{CHH}$ but not in AD. Furthermore, the $\mathrm{CHH}$ founder mutation $(70 \mathrm{~A} \rightarrow \mathrm{G})$ affected both rRNA and mRNA processing, whereas mutations resulting in $\mathrm{AD}$ (Table 1) affected ribosomal assembly but not $C L B 2$ mRNA levels [20]. This has led to the suggestion that the disruption of $C L B 2$ mRNA levels by mutation in 
the $R M R P$ gene is the main cause of bone marrow failure in $\mathrm{CHH}$ patients [20].

As RNase MRP has three RNA-processing activities (Figure 1), the deficiency of its enzyme activity caused by either mutated nucleotides or an altered promoter region in the $R M R P$ gene would negatively affect all three physiological functions. Thus, it is our belief that the pathogenesis of $\mathrm{CHH}$ is likely to be related to all three known functions of RNase MRP, not just one or two. Six CHH-relevant mutations (Figure 2) on the MRP RNA are also found in other diseases, 4C $\rightarrow \mathrm{U}(\mathrm{OS}), 194 \_195 \mathrm{ins} \mathrm{U}$ and $63 \mathrm{C} \rightarrow \mathrm{U}(\mathrm{KD})$, and $70 \mathrm{~A} \rightarrow \mathrm{G}, 195 \mathrm{C} \rightarrow \mathrm{U}$ and $238 \mathrm{C} \rightarrow \mathrm{U}(\mathrm{MDWH})$. Single nucleotide mutations at the same position $(14 \mathrm{G} \rightarrow \mathrm{U}$ and $14 \mathrm{G} \rightarrow \mathrm{A}$ ) are found in both $\mathrm{CHH}$ and MDWH (Figure 2). Yet, no identical homozygous or heterozygous mutations have been found in more than one genetic disorders. It is plausible that different mutations of the RMRP gene lead to diverse genetic disorders. However, given that $\mathrm{CHH}$ is both pleiotropic and variable in clinical severity and not all CHH patients have mutated $R M R P$ genes [15], that the MRP RNA is a noncoding RNA and the reconstitution of the RNase MRP complex with the RNA and its protein components is not yet accomplished, and that there is a very limited number of clinical cases of genetic disorders other than CHH (Table 1), it is very difficult, if not impossible at this stage, to fully evaluate the pathogenicity of $R M R P$ gene on $\mathrm{CHH}$ and other skeletal dysplasias. Several laboratories have tried to construct a gene-knockin mouse model of CHH (Dr Cynthia L Jackson and an NIH group; personal communication), although no success has been reported thus far. Depletion of Rpp1, an essential protein component of RNase MRP, led to dysregulated expression of a large number of genes in yeast [26]. This indicates that RNase MRP may perform other functions beyond those that have been revealed (Figure 1). The functional complication of RNase MRP would certainly increase the complexity to assess the phenotype/genotype relationship of $R M R P$ mutations in various genetic disorders.

\section{RNase MRP and RNase $P$}

RNase MRP is closely related to RNase P, a ubiquitous ribonucleoprotein that cleaves precursor tRNA transcripts to produce mature $5^{\prime}$ termini [27]. In both yeast and human cells, each enzyme consists of one RNA subunit and several proteins $[6,7]$. The RNA subunits of both ribonucleoproteins can be folded into similar secondary structures and they share most of the protein subunits, indicating that they are both structurally and evolutionarily related $[6,7]$.

The Schmid type of MCD (MCDS, OMIM, \#156500) is generally caused by mutations in the COL10A1-encoding type X collagen of cartilage. MCDS is characterized by short stature, bowed legs, coax vara, and specific metaphyseal changes, which are visible in radiographs. As the clinical features of MCDS and $\mathrm{CHH}$ are similar to each other, Ridanpää et al. [28] performed a study on the genes for the RNA subunit of both RNase MRP and RNase P (H1 RNA) among 20 patients who had been diagnosed with MCDS but had no mutations in COL10A1. They found two patients homozygous for the $70 \mathrm{~A} \rightarrow \mathrm{G}$ change in $R M R P$, which is widely known as the major mutation in patients with $\mathrm{CHH}$. Yet, these two patients were not diagnosed with $\mathrm{CHH}$, based on the distinction of growth failure at birth and during the first year of life (present in $\mathrm{CHH}$ but not in MCDS) and subtle radiographic metaphyseal changes (most prominent in knees in $\mathrm{CHH}$, patients have short hands; yet most prominent in hips in MCDS, patients have normal hands) [28]. As RNase P and RNase MRP are closely related, Ridanpää et al. reasoned that the H1 RNA, the RNA subunit of RNase P, may be mutated in these patients. The authors did find base substitution polymorphisms (including a mutation at position 129G, which is evolutionally conserved) in the H1 RNA gene in MCDS patients; yet these base substitutions were also found in control patients albeit with a lower frequency. As the sample size was limited and the RNase P activity corresponding to these mutations was not examined, the authors were unable to show conclusive evidence for causative mutations in $\mathrm{H} 1$ RNA in patients with MCDS, and the role of RNase P in skeletal dysplasias remains unclear.

In conclusion, the RNase MRP RNA is the first nuclearencoded RNA in which mutations have been found to lead to human diseases. Yet the precise relationship of $\mathrm{CHH}$ and other genetic diseases with the three disclosed functions of RNase MRP in three cellular compartments (mitochondria, nucleoli, and cytoplasm) requires further investigation.

\section{Acknowledgments}

Research on RNase MRP in Li Y's laboratory is supported by a pilot study grant for Systems Biology from the Center for Genetics and Molecular Medicine, University of Louisville. Li Y is supported by a Beginning-GrantIn-Aid grant from the American Heart Association. Li Y is grateful to fantastic colleagues Drs Mark D Brennan, Marlene C Steffen, and Yanglong Zhu for critical reading of the manuscript.

\section{References}

1 McKusick VA. Mendelian inheritance in man. A catalog of human genes and genetic disorders. Baltimore: Johns Hopkins University Press, 1998; Online Mendelian Inheritance in Man (OMIM) is hosted at http://www.ncbi.nlm.nih.gov/omim/. 
2 Chang DD, Clayton DA. Mouse RNAase MRP RNA is encoded by a nuclear gene and contains a decamer sequence complementary to a conserved region of mitochondrial RNA substrate. Cell 1989; 56:131-139.

3 Lygerou Z, Allmang C, Tollervey D, Seraphin B. Accurate processing of a eukaryotic precursor ribosomal RNA by ribonuclease MRP in vitro. Science 1996; 272:268-270.

4 Schmitt ME, Clayton DA. Nuclear RNase MRP is required for correct processing of pre-5.8S rRNA in Saccharomyces cerevisiae. Mol Cell Biol 1993; 13:7935-7941.

5 Li K, Smagula CS, Parsons WJ, et al. Subcellular partitioning of MRP RNA assessed by ultrastructural and biochemical analysis. J Cell Biol 1994; 124:871-882.

6 van Eenennaam H, Jarrous N, van Venrooij WJ, Pruijn GJ. Architecture and function of the human endonucleases RNase $P$ and RNase MRP. IUBMB Life 2000; 49:265-272.

7 Chamberlain JR, Lee Y, Lane WS, Engelke DR. Purification and characterization of the nuclear RNase P holoenzyme complex reveals extensive subunit overlap with RNase MRP. Genes Dev 1998; 12:1678-1690.

8 Gill T, Aulds J, Schmitt ME. A specialized processing body that is temporally and asymmetrically regulated during the cell cycle in Saccharomyces cerevisiae. J Cell Biol 2006; 173:35-45.

9 Gill T, Cai T, Aulds J, Wierzbicki S, Schmitt ME. RNase MRP cleaves the CLB2 mRNA to promote cell cycle progression: novel method of mRNA degradation. Mol Cell Biol 2004; 24:945953.

10 McKusick VA, Eldridge R, Hostetler JA, Ruangwit U, Egeland JA. Dwarfism in the Amish. Ii. Cartilage-hair hypoplasia. Bull Johns Hopkins Hosp 1965; 116:285-326.

11 Ridanpää M, Sistonen P, Rockas S, et al. Worldwide mutation spectrum in cartilage-hair hypoplasia: ancient founder origin of the major $70 \mathrm{~A} \rightarrow \mathrm{G}$ mutation of the untranslated RMRP. Eur J Hum Genet 2002; 10:439-447.

12 Ridanpää M, van Eenennaam H, Pelin K, et al. Mutations in the RNA component of RNase MRP cause a pleiotropic human disease, cartilage-hair hypoplasia. Cell 2001; 104:195-203.

13 Bonafé L, Schmitt K, Eich G, Giedion A, Superti-Furga A. RMRP gene sequence analysis confirms a cartilage-hair hypoplasia variant with only skeletal manifestations and reveals a high density of single-nucleotide polymorphisms. Clin Genet 2002; 61:146151.

14 Hirose Y, Nakashima E, Ohashi H, et al. Identification of novel RMRP mutations and specific founder haplotypes in Japanese patients with cartilage-hair hypoplasia. J Hum Genet 2006; 51:706-710.

15 Hermanns P, Tran A, Munivez E, et al. RMRP mutations in cartilage-hair hypoplasia. Am J Med Genet A 2006 140:2121-2130.

16 Nakashima E, Mabuchi A, Kashimada K, et al. RMRP mutations in Japanese patients with cartilage-hair hypoplasia. Am J Med
Genet A 2003; 123:253-256.

17 Ridanpää M, Jain P, McKusick VA, Francomano CA, Kaitila I. The major mutation in the RMRP gene causing $\mathrm{CHH}$ among the Amish is the same as that found in most Finnish cases. Am J Med Genet C Semin Med Genet 2003; 121:81-83.

18 Hermanns P, Bertuch AA, Bertin TK, et al. Consequences of mutations in the non-coding RMRP RNA in cartilage-hair hypoplasia. Hum Mol Genet 2005; 14:3723-3740.

19 Bonafé L, Dermitzakis ET, Unger S, et al. Evolutionary comparison provides evidence for pathogenicity of RMRP mutations. PLoS Genet 2005; 1:e47.

20 Thiel CT, Horn D, Zabel B, et al. Severely incapacitating mutations in patients with extreme short stature identify RNAprocessing endoribonuclease RMRP as an essential cell growth regulator. Am J Hum Genet 2005; 77:795-806.

21 Roifman CM, Gu Y, Cohen A. Mutations in the RNA component of RNase mitochondrial RNA processing might cause Omenn syndrome. J Allergy Clin Immunol 2006; 117:897-903.

22 Kuijpers TW, Ridanpää M, Peters M, et al. Short-limbed dwarfism with bowing, combined immune deficiency, and late onset aplastic anaemia caused by novel mutations in the RMPR gene. J Med Genet 2003; 40:761-766.

23 Prasad C, Cramer BC, Pushpanathan C, Crowley MC, Ives EJ. Kyphomelic dysplasia: a rare form of semilethal skeletal dysplasia. Clin Genet 2000; 58:390-395.

24 Bommer U-A, Stahl J, Henske A, Lutsch G, Bielka H. Identification of proteins of the $40 \mathrm{~S}$ ribosomal subunit involved in interaction with initiation factor eIF-2 in the quaternary initiation complex by means of monospecific antibodies. FEBS Lett 1988; 233:114-118.

25 Abou Elela S, Nazar RN. Role of the 5.8S rRNA in ribosome translocation. Nucleic Acids Res 1997; 25:1788-1794.

26 Samanta MP, Tongprasit W, Sethi H, Chin CS, Stolc V. Global identification of noncoding RNAs in Saccharomyces cerevisiae by modulating an essential RNA processing pathway. Proc Natl Acad Sci USA 2006; 103:4192-4197.

27 Altman S, Kirsebom L. Ribonuclease P. In: Gesteland R, Cech T, Atkins J, eds. The RNA world. New York: Cold Spring Harbor Laboratory Press, 1999:351-380.

28 Ridanpää M, Ward LM, Rockas S, et al. Genetic changes in the RNA components of RNase MRP and RNase P in Schmid metaphyseal chondrodysplasia. J Med Genet 2003; 40:741-746.

29 Shadel GS, Clayton DA. Mitochondrial DNA maintenance in vertebrates. Annu Rev Biochem 1997; 66:409-435.

30 Venema J, Tollervey D. Ribosome synthesis in Saccharomyces cerevisiae. Annu Rev Genet 1999; 33:261-311.

31 Cai T, Aulds J, Gill T, Cerio M, Schmitt ME. The Saccharomyces cerevisiae RNase mitochondrial RNA processing is critical for cell cycle progression at the end of mitosis. Genetics 2002; 161:1029-1042. 